\title{
A New Nature-inspired Algorithm for Load Balancing
}

\author{
Xiang Feng \\ Department of Computer Science \\ East China University of \\ Science and Technology \\ Shanghai, China 200237 \\ Email: xfeng\{@ecust.edu.cn, @cs.hku.hk\}
}

\author{
Francis C.M. Lau \\ Department of Computer Science \\ The University of \\ Hong Kong, Hong Kong \\ Email: fcmlau@cs.hku.hk
}

\author{
Dianxun Shuai \\ Department of Computer Science \\ East China University of \\ Science and Technology \\ Shanghai, China 200237 \\ Email: shdx411022@online.sh.cn
}

\begin{abstract}
The classical Load Balancing Problem (LBP) is to map tasks to processors so as to minimize the maximum load. Solving the LBP successfully would lead to better utilization of resources and better performance. The LBP has been proven to be NP-hard, thus generating the exact solutions in a tractable amount of time becomes infeasible when the problems become large. We present a new nature-inspired approximation algorithm based on the Particle Mechanics (PM) model to compute in parallel approximate efficient solutions for LBPs. Just like other Nature-inspired Algorithms (NAs) drawing from observations of physical processes that occur in nature, the PM algorithm is inspired by physical models of particle kinematics and dynamics. The PM algorithm maps the classical LBP to the movement of particles in a force field by a corresponding mathematical model in which all particles move according to certain defined rules until reaching a stable state. By anti-mapping the stable state, the solution to LBP can be obtained.

Index Terms-Load balancing, approximation algorithm, nature-inspired algorithm, particle mechanics model, distributed and parallel algorithm.
\end{abstract}

\section{INTRODUCTION}

An increasingly large number of scientific pursuits use computational resources as their backbone. Parallelism at the scale of tens of thousands of processors is being seen. It is imperative that more and better techniques for efficient utilization of the large scale parallel machines be developed. The main resources in a large parallel machine are its compute nodes and the interconnection network; both must be utilized efficiently. Load balancing is an age-old but effective technique for achieving better utilization of computational resources in a parallel environment.

The classical Load Balancing Problem (LBP) is to map tasks to processors so as to minimize the maximum load. Of course, there are also a variety of other problems involving task migration to balance load. The systems community has shown considerable interest in the problem of process migration in the context of scheduling tasks on multiple processors. Some of the benefits derived from process migration include better resource utilization and better overall performance.

The LBP has been proved to be NP-hard [1], which naturally means that we are unable to deliver an exact solution to the problem in a reasonable amount of time when the problem gets large. For instance, if we have 2000 tasks and 10 processors that we could use, we have $10^{2000}$ configurations to enumerate and compare for the LBP. While solving these problems is not feasible under such a situation, approximation algorithms may possibly be implemented to achieve a reasonable estimate within a fixed error ratio in a reasonable amount of time.

Recently, several load balancing assignment policies have been proposed [2-5]). According to two main strategies, these policies attempt to balance the load among the back-end servers:

1) balancing the amount of workload at the back-end servers, and

2) balancing the number of jobs being processed by the back-end servers.

In this paper, we present a nature-inspired approximation algorithm based on the Particle Mechanics (PM) model to compute in parallel approximate efficient solutions for LBPs.

\section{PROBLEM MODEL FOR LBP}

\section{The Load Balancing Problem (LBP)}

Given:

- A network of processors, $A_{j}$, and their processing capacities $x_{j}$ (in task units per second). There is a communication channel between any two processors. A processor is characterized by the attribute: its "remaining workload", $w_{j}$ (in task units).

- A set of tasks, $T_{i}$, that are spread across these processors in a given "initial mapping"; a task is characterizied by the attribute: its "size", $s_{i}$ (in bytes).

Goal: To map the tasks to the processors so that the execution time which is the maximum of the individual execution times after the mapping is minimized.

The main notations in any LBP instance are shown in Table I.

We can formalize LBP using a matrix $\Lambda$ (see Table II).

In Table II, the task mapping variable, $r_{i j}$, is the key of the LBP. If an algorithm can compute and update $r_{i j}$ in parallel without any information exchange, the algorithm has a chance to solve the LBP in parallel efficiently. 
TABLE II

THE REPRESENTATION MATRIX $\Lambda$ OF LBP, WITH PROCESSORS (A) AND TASKS (T)

\begin{tabular}{||c|ccccc|c||}
\hline \hline & $A_{1}$ & $\cdots$ & $A_{j}$ & $\cdots$ & $A_{n}$ & $s_{i}$ \\
$T_{1}$ & $r_{11}, e_{11}$ & $\cdots$ & $r_{1 j}, e_{1 j}$ & $\cdots$ & $r_{1 n}, e_{1 n}$ & $s_{1}=\sum_{j=1}^{n} r_{1 j}$ \\
$\vdots$ & $\vdots$ & & $\vdots$ & & $\vdots$ & $\vdots$ \\
$T_{i}$ & $r_{i 1}, e_{i 1}$ & $\cdots$ & $r_{i j}, e_{i j}$ & $\cdots$ & $r_{i n}, e_{i n}$ & $s_{i}=\sum_{j=1}^{n} r_{i j}$ \\
$\vdots$ & $\vdots$ & $\vdots$ & & $\vdots$ & $\vdots$ \\
$T_{m}$ & $r_{m 1}, e_{m 1}$ & $\cdots$ & $r_{m j}, e_{m j}$ & $\cdots$ & $r_{m n}, e_{m n}$ & $s_{m}=\sum_{j=1}^{n} r_{m j}$ \\
\hline$w_{j}$ & $w_{1}=\sum_{i=1}^{m} r_{i 1}$ & $\cdots$ & $w_{j}=\sum_{i=1}^{m} r_{i j}$ & $\cdots$ & $w_{n}=\sum_{i=1}^{m} r_{i n}$ & $\sum_{j=1}^{n} w_{j}=\sum_{i=1}^{m} s_{i}$ \\
\hline \hline
\end{tabular}

TABLE I

THE MAIN NOTATIONS IN LBP

\begin{tabular}{||c|c||}
\hline \hline Notations & Meanings \\
\hline$A_{j}$ & The -th processor $(j=\overline{1, n})$ \\
\hline$T_{i}$ & Tht task $(i=\overline{1, m})$ \\
\hline$r_{i j}$ & The subtask of task $T_{i}$ that is mapped to processor $A_{j}$ \\
\hline$x_{j}$ & The processing capacities of processor $A_{j}$ \\
\hline$w_{j}$ & The remaining workload of processor $A_{j}$ \\
\hline$s_{i}$ & The size of task $T_{i}$ \\
\hline$e_{i j}$ & The execution time of $r_{i j}, e_{i j}=r_{i j} / x_{j}$ \\
\hline \hline
\end{tabular}

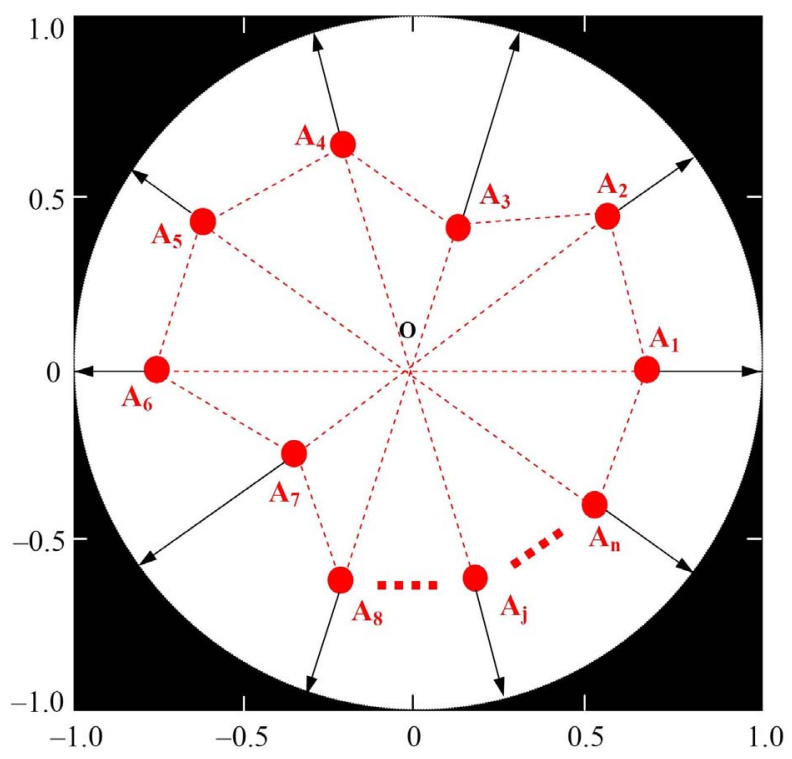

Fig. 1. The architecture of a particle mechanics model for LBP

\section{ThE MOTIVATION AND ARChITECTURE OF PM MODEL}

Figure 1 illustrates the physical model of the PM model. Here we introduce two key concepts (particles and force-field) of our PM model to describe and model the optimization of the LBP. We treat processors as particles, which are surrounded by a circumferential force-field. The force-field can make the particles' minimal utility increase (which will be proved in next section). The analogical relation between the PM model
TABLE III

THE ANALOGICAL RELATION BETWEEN PM MODEL AND LBP

\begin{tabular}{||c|c||}
\hline \hline In PM model & In LBP \\
\hline Particles & Processors \\
\hline Force-field & Max-min fairness balancing of LBP \\
\hline $\begin{array}{c}\text { Radial distance between } \\
\text { the origin and a particle }\end{array}$ & The processor's personal utility \\
\hline $\begin{array}{c}\text { Radial distance between a } \\
\text { particle and the circumference }\end{array}$ & $\begin{array}{c}\text { The standardization of the } \\
\text { execution times of the processor }\end{array}$ \\
\hline Gravitation of force-field & Make LBP optimize \\
\hline Motion of all processors & The process of LBP optimization \\
\hline $\begin{array}{c}\text { Forces that force-field } \\
\text { exerts on particles }\end{array}$ & $\begin{array}{c}\text { The trend and degree of processors } \\
\text { will be optimized }\end{array}$ \\
\hline
\end{tabular}

and the LBP can be seen in Table III.

In the PM model, all particles (processors) are evenly distributed at an even radian. Each particle is being exerted upon simultaneously by the force-field of the circumference surrounding the particles. The radial distance between a particle and the origin represents the corresponding particle's personal utility - that is, a processor's profit according to the current situation of the LBP. Based on the LBP, the shorter the execution times of a processor, the larger the processor's personal utility. The LBP aims to minimize the maximum of the individual execution times of all processors. The force-field will make the particle that is the closest from the origin move out along the radius - that is, to maximize the minimum of all particles' (processors') utility, the corresponding particle in force-field will try to move as close as possible to the circumference surrounding it.

\section{THE MATHEMATICAL MODEL AND CORRESPONDING ALGORITHM OF PM}

We now examine the evolutionary model that can mathematically describe the PM's physical model for the LBP.

We can obtain the execution times of every $r_{i j}$ as

$$
e_{i j}(t)=\frac{r_{i j}(t)}{x_{j}(t)}
$$

and the execution times of processor $A_{j}$ as 


$$
e_{j}(t)=\sum_{i=1}^{m} \frac{r_{i j}(t)}{x_{j}(t)}
$$

Because $1-e^{-x}$ is a monotone increasing function and between 0 and 1 , we choose the function to standardize the execution times as

$$
E_{j}(t)=1-\exp \left[-e_{j}(t)\right]=1-\exp \left[-\sum_{i=1}^{m} \frac{r_{i j}(t)}{x_{j}(t)}\right]
$$

where $E_{j}$ represents the radial distance from particle $A_{j}$ to the circumference in force-field. The radius of the force-field will always be 1 and $E_{j}$ will be in $[0,1]$.

We define the personal utility function for particle (processor) $A_{j} \in\left\{A_{1}, \cdots, A_{n}\right\}$ as

$$
u_{j}(t)=1-E_{j}(t)=\exp \left[-\sum_{i=1}^{m} \frac{r_{i j}(t)}{x_{j}(t)}\right]
$$

where $u_{j}$ represents the radial distance from the origin to particle $A_{j}$ in the force-field. The longer the execution times of a processor, the smaller the processor's personal utility, and the corresponding particle will be closer to the origin in the force-field.

We define the potential energy function of the force-field as

$$
P(t)=k^{2} \ln \sum_{j=1}^{n} \exp \left[\frac{E_{j}^{2}(t)}{2 k^{2}}\right]
$$

Theorem 1: In equation 5, if $k$ is very small, the decrease of the potential energy $P(t)$ of the force-field will cause a decrease of the processors' maximal execution times.

Proof. Supposing that $H(t)=\max _{j} E_{j}^{2}(t)$, we have

$$
\left[e^{\frac{H(t)}{2 k^{2}}}\right]^{2 k^{2}} \leq\left[\sum_{j=1}^{n} e^{\frac{E_{j}^{2}(t)}{2 k^{2}}}\right]^{2 k^{2}} \leq\left[n e^{\frac{H(t)}{2 k^{2}}}\right]^{2 k^{2}}
$$

Taking the logarithm for both sides of Eq. 6 leads to

$$
H(t) \leq 2 k^{2} \ln \left[\sum_{j=1}^{n} e^{\frac{E_{j}^{2}(t)}{2 k^{2}}}\right] \leq H(t)+2 k^{2} \ln n
$$

Because $n$ is constant and $k$ is very small, we have

$$
H(t)=\max _{j} E_{j}^{2}(t) \approx 2 k^{2} \ln \left[\sum_{j=1}^{n} e^{\frac{E_{j}^{2}(t)}{2 k^{2}}}\right]=2 P(t)
$$

The potential energy function $P(t)$ of the force-field at time $t$ turns out to represent the maximal execution times among $E_{j}(t), j=1, \cdots, n$. So, decreasing $P(t)$ amounts to decreasing the processors' maximal execution times.

We define the dynamic equation of subtask $r_{i j}(t)$ in the PM model as

$$
r_{i j}(t+1)=r_{i j}(t)+\triangle r_{i j}(t)
$$

The dynamic equation is seen as the "PM evolution", which manipulates the update and iteration of $r_{i j}$ until an equilibrium is reached. By the PM algorithm, $r_{i j}$ can be computed and updated in parallel without any information exchange, which is the foundation of the algorithm's parallelism.

The most important related factor that influences the update and iteration of $r_{i j}$ is the potential energy function $P(t)$. According to "differential equation theory", a variable's increment to make it minimum is equal to the sum of negative items from related factors differentiating the variable. Thus we define the first item of $\triangle r_{i j}(t)$ as

$$
\triangle r_{i j}(t+1)=-\lambda_{1} \frac{\partial P(t)}{\partial r_{i j}(t)}
$$

where $0<\lambda_{1}<1$.

Theorem 2: The update and iteration of $r_{i j}$ according to Eq. 8 will always cause a decrease of the processors' maximal execution times.

Proof. Consider the effect of only the potential energy $P(t)$ on $r_{i j}(t+1)$; namely, let $\triangle r_{i j}(t+1)$ be $-\lambda_{1} \frac{\partial P(t)}{\partial r_{i j}(t)}$ (Eq. 8).

We determine the increment of the potential energy $P(t)$ in the unit time period as follows:

$$
\triangle P(t)=\frac{\partial P(t)}{\partial r_{i j}} \frac{d r_{i j}}{d t} \approx \frac{\partial P(t)}{\partial r_{i j}} \triangle r_{i j}=-\lambda_{1}\left\|\frac{\partial P(t)}{\partial r_{i j}}\right\|^{2} \leq 0
$$

So, the update and iteration of $r_{i j}(t)$ according to Eq. 8 will reduce the potential energy $P(t)$, with the intension strength being $\lambda_{1}$. By Theorem 1, the conclusion thus is straightforward.

The other important related factor that influences the update and iteration of $r_{i j}$ is the utility function $u_{j}(t)$. Because $u_{j}(t)$ needs to maximize, we use its opposite function $E_{j}(t)$ to define the second item of $\triangle r_{i j}(t+1)$ as

$$
\triangle r_{i j}(t+1)=-\lambda_{2} \frac{\partial E_{j}(t)}{\partial r_{i j}(t)}
$$

Theorem 3: The update and iteration of $r_{i j}$ according to Eq. 9 will always cause a decrease of the processors' execution times.

Proof. Consider the effect of only the execution times function $E_{j}(t)$ on $r_{i j}(t+1)$; namely, let $\triangle r_{i j}(t+1)$ be $-\lambda_{1} \frac{\partial P(t)}{\partial r_{i j}(t)}-\lambda_{2} \frac{\partial E_{j}(t)}{\partial r_{i j}(t)}$ (Eqs. 8, 9).

Then, the changing rate of the personal execution times of processor $A_{j}$ is equal to

$$
\begin{aligned}
\triangle E_{j}(t) & =\frac{d E_{j}}{d t}=\frac{\partial E_{j}}{\partial r_{i j}} \frac{d r_{i j}}{d t} \approx \frac{\partial E_{j}}{\partial r_{i j}} \Delta r_{i j} \\
& =\frac{\partial E_{j}}{\partial r_{i j}}\left[-\lambda_{1} \frac{\partial P(t)}{\partial r_{i j}}-\lambda_{2} \frac{\partial E_{j}}{\partial r_{i j}}\right] \\
& =\frac{\partial E_{j}}{\partial r_{i j}}\left[-\lambda_{1} \frac{\partial P(t)}{\partial E_{j}} \frac{\partial E_{j}}{\partial r_{i j}}-\lambda_{2} \frac{\partial E_{j}}{\partial r_{i j}}\right] \\
& =-\left[\lambda_{1} \frac{\partial P(t)}{\partial E_{j}}+\lambda_{2}\right]\left\|\frac{\partial E_{j}}{\partial r_{i j}}\right\|^{2}
\end{aligned}
$$

where 


$$
\frac{\partial P(t)}{\partial E_{j}}=E_{j} \frac{\exp \left(\frac{E_{j}^{2}}{2 k^{2}}\right)}{\sum_{j=1}^{n} \exp \left(\frac{E_{j}^{2}}{2 k^{2}}\right)} \geq 0
$$

Thus

$$
\triangle E_{j}(t) \leq 0
$$

So, the personal execution times of processor $A_{j}$ will decrease. In the PM physical model, by the theorem, we can conclude that all particles will try to move as close as possible to the circumference along their own radial orbits.

Combining Eq. 8 and Eq. 9, we have

$$
\triangle r_{i j}(t+1)=-\lambda_{1} \frac{\partial P(t)}{\partial r_{i j}(t)}-\lambda_{2} \frac{\partial E_{j}(t)}{\partial r_{i j}(t)}
$$

In order to satisfy the constraints of the LBP, $r_{i j}$ will be dealt with using the following two steps in the parallel computing process.

- Nonnegativity: If $\min _{i, j}<0$, then let $r_{i j}=r_{i j}-\min _{i, j} r_{i j}$.

- Normalization: Let $r_{i j}=\frac{r_{i j}}{\sum_{j=1}^{n} r_{i j}}$, in order to map all tasks to processors; that is, $\sum_{j=1}^{n} r_{i j}=1, i=1, \cdots, m$.

We can obtain the radial velocity of particle $A_{j}$ along its radial orbit to the circumference of the force-field by the equation

$$
v_{j}=\frac{d E_{j}}{d t}=\sum_{i=1}^{m} \frac{\partial E_{j}}{\partial r_{i j}} \frac{d r_{i j}}{d t}=\frac{u_{j}}{x_{j}} \sum_{i=1}^{m} \frac{d r_{i j}}{d t} \approx \frac{u_{j}}{x_{j}} \sum_{i=1}^{m} \triangle r_{i j}
$$

When all $v_{j}(j=1, \cdots, n)$ are equal to 0 , the PM model reaches a stable state, and the solution to LBP is obtained.

\section{The parallel PM algorithm for LBP}

\section{Input:

$$
s_{i}, x_{j}
$$

1. Initialization:

$$
t \leftarrow 0
$$

$$
\triangle t, \lambda_{1}, \lambda_{2}, r_{i j}(t)
$$

2. $\quad$ while $\left(v_{j}(t) \neq 0\right)$ do

$$
t \leftarrow t+1
$$

Compute $E_{j}(t)$ according to equation 3

Compute $\triangle r_{i j}(t)$ according to equation 10

$r_{i j}(t) \leftarrow r_{i j}(t-1)+\triangle r_{i j}(t)$

Compute $v_{j}(t)$ according to equation 11

$$
\begin{aligned}
& \text { If } \min _{i, j}(t)<0 \text {, then } r_{i j}(t) \leftarrow r_{i j}(t)-\min _{i, j} r_{i j}(t) \\
& r_{i j}(t) \leftarrow \frac{r_{i j}(t)}{\sum_{j=1}^{n} r_{i j}(t)}
\end{aligned}
$$

At the end, when an equilibrium is reached, $r_{i j}=r_{i j} \cdot s_{i}$ is the solution to LBP.

\section{CONVERGENCE AND PARAMETERS ANALYSIS}

\section{A. Convergence analysis}

In this section, we construct a Lyapunov function, which is an energy-related positive definite function. We can judge the stability of the PM model by analyzing if the Lyapunov function monotonically decreases with the elapsing time.

Lyapunov second theorem on stability Consider a function $L(X)$ such that

- $L(X)>0$ (positive definite);

- $d L(X(t)) / d t<0$ (negative definite).

Then $L(X(t))$ is called a Lyapunov function candidate and $X$ is asymptotically stable in the sense of Lyapunov.

It is easier to visualize this method of analysis by considering the energy of the PM model. If the PM model loses energy over time, then eventually the model must grind to a halt and reach some final resting state. This final state is called the stable equilibrium state.

Theorem 4: If the condition (Eq. 12) remains valid, then the PM model will converge to a stable equilibrium state.

Proof. For the physical PM model, we define a Lyapunov function $L\left(r_{i j}(t)\right)$ as

$$
L\left(r_{i j}(t)\right) \triangleq \sum_{i, j} r_{i j}(t)+2 \lambda_{2} \sum_{j=1}^{n} \int_{0}^{t} \frac{u_{j}(y)}{x_{j}} d y
$$

Obviously, $L\left(r_{i j}(t)\right)>0$.

$$
\begin{aligned}
\because \frac{d E_{j}(t)}{d r_{i j}(t)} & =\frac{u_{j}(t)}{x_{j}} \\
\frac{d P(t)}{d r_{i j}(t)} & =\frac{\partial P(t)}{\partial E_{j}(t)} \frac{d E_{j}(t)}{d r_{i j}(t)}=\frac{E_{j} \cdot u_{j}}{x_{j}} \cdot \frac{\exp \left(\frac{E_{j}^{2}(t)}{2 k^{2}}\right)}{\sum_{j=1}^{n} \exp \left(\frac{E_{j}^{2}(t)}{2 k^{2}}\right)}
\end{aligned}
$$

$$
\begin{aligned}
\triangle r_{i j}(t) & =-\lambda_{1} \frac{\partial P(t)}{\partial r_{i j}(t)}-\lambda_{2} \frac{\partial E_{j}(t)}{\partial r_{i j}(t)} \\
& =-\frac{u_{j}(t)}{x_{j}}\left[\lambda_{1} \frac{E_{j}(t) \exp \left(\frac{E_{j}^{2}(t)}{2 k^{2}}\right)}{\sum_{j=1}^{n} \exp \left(\frac{E_{j}^{2}(t)}{2 k^{2}}\right)}+\lambda_{2}\right] \\
\therefore \frac{d L(t)}{d t} & =\frac{d r_{i j}(t)}{d t}+2 \lambda_{2} \frac{u_{j}(t)}{x_{j}}=\Delta r_{i j}(t)+2 \lambda_{2} \frac{u_{j}(t)}{x_{j}} \\
& =\frac{u_{j}(t)}{x_{j}}\left[-\lambda_{1} \frac{E_{j}(t) \exp \left(\frac{E_{j}^{2}(t)}{2 k^{2}}\right)}{\sum_{j=1}^{n} \exp \left(\frac{E_{j}^{2}(t)}{2 k^{2}}\right)}+\lambda_{2}\right]
\end{aligned}
$$

Because $\frac{u_{j}(t)}{x_{j}}>0$, if the following Eq. 12 remains valid, then $d L(t) / d t<0$.

$$
\lambda_{2}<\lambda_{1} \frac{E_{j}(t) \exp \left(\frac{E_{j}^{2}(t)}{2 k^{2}}\right)}{\sum_{j=1}^{n} \exp \left(\frac{E_{j}^{2}(t)}{2 k^{2}}\right)}
$$


Based on the Lyapunov second theorem on stability, as long as we properly select the parameters $\lambda_{1}, \lambda_{2}$ according to Eq. 12 , the convergence and stability can be guaranteed. That is, when $t \rightarrow \infty$, then all $r_{i j}(t) \rightarrow r_{i j}$ (constants).

\section{B. Parameters analysis}

There are only three parameters in the PM model and algorithm, $k$ in Eq. 5 and $\lambda_{1}, \lambda_{2}$ in Eq. 10 .

$k$ represents the strength of the gravitational force in the force-field. The larger $k$ is, the faster the particles would move away from the origin along their radial orbits; hence, $k$ influences the convergence speed of the PM algorithm. As required in Theorem $1, k$ must be small. Usually, $0<k<1$.

$$
\because 0<\frac{E_{j}(t) \exp \left(\frac{E_{j}^{2}(t)}{2 k^{2}}\right)}{\sum_{j=1}^{n} \exp \left(\frac{E_{j}^{2}(t)}{2 k^{2}}\right)} \leq \frac{\exp \left(\frac{E_{j}^{2}(t)}{2 k^{2}}\right)}{\sum_{j=1}^{n} \exp \left(\frac{E_{j}^{2}(t)}{2 k^{2}}\right)} \approx \frac{1}{n}<1
$$

According to Eq. 12, $\lambda_{2}$ should be much smaller than $\lambda_{1}-$ that is,

$$
\lambda_{2}<\frac{\lambda_{1}}{n}
$$

where $n$ is the number of processors in the LBP.

\section{Simulations}

Simulations of the LBP verify our PM algorithm's advantages in terms of parallelism and convergence speed to an optimal solution. The simulation used these parameters: $k=0.8, \lambda_{1}=0.9, \lambda_{2}=\frac{0.9}{n}$.

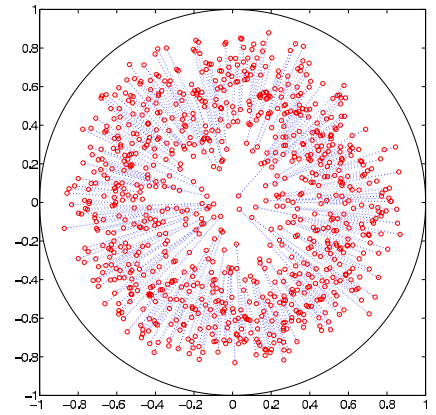

(a) $t=0$

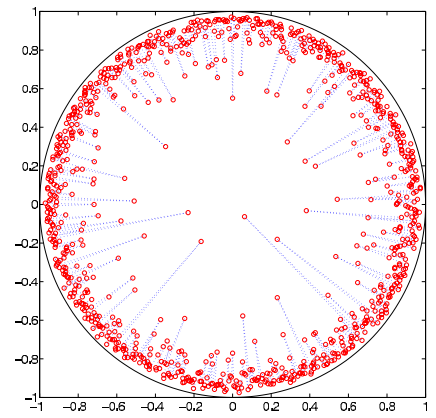

(d) $t=600$

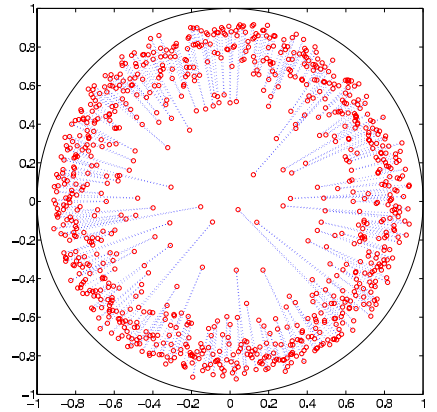

(b) $t=300$

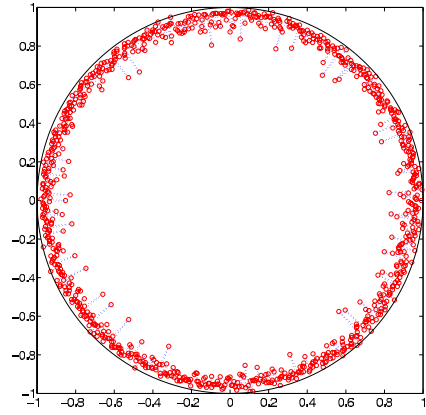

(e) $t=851$
Fig. 2. The trajectories of particles using the PM algorithm for the LBP $(n=900)$
Because the parallel computation of $r_{i j}(t)$ is the foundation of the algorithm's parallelism, the PM algorithm is scalable. When the number of processors $(n)$ in the LBP is very large (e.g., $n$ is more than 10000), the PM algorithm can still perform well. Here we will give the experimental results for the LBP $(n=900)$ in Figure 2.

As shown in Figure 2(a), most of particles are far from the circumference-that is, most $E_{j}$ are large, which represent most execution times $e_{j}$ of processors are long. In Figure 2(b) and (c), by some iterations, particles move away from the origin along their radial orbits, which represents that the execution times of most of the processors are being shortened. In Figure 2(d), at the end of the PM algorithm, particles move as close as possible to the circumference in the force-field, which represents that all execution times of processors are balanced and substantially shortened.

\section{CONCLUSION}

In this paper, we propose the PM model and algorithm for the LBP. The PM algorithm is inspired by the physical model of particle dynamics. Our future research direction include solving the Load Rebalancing Problem (LRP), where progresses of the computation may dynamically alter the load distribution, and is therefore more difficult than the LBP. We will conceive a physical Water Flow model to model the LRP, and its corresponding mathematical model and algorithm.

\section{Acknowledgment}

This project is supported in part by the Hong Kong General Research Fund (HKU 713708E).

\section{REFERENCES}

[1] Kleinberg G. and Tardos E. Algorithm Design, Cornell University, Spring 2004.

[2] P. Bruckner, Scheduling Algorithms, third ed., Springer-Verlag, 2001.

[3] M. Pinedo, Scheduling: Theory, Algorithms, and Systems, Prentice Hall, 2002.

[4] S.M. Ross, Probability Models for Computer Science, Academic Press, 2002.

[5] V. Ungureanu, B. Melamed, M. Katehakis, P.G. Bradford, Deferred assignment scheduling in cluster-based servers, Cluster Computing 9 (1) (2006). 